\title{
Experimental study of spatiotemporally localized surface gravity water waves
}

\author{
A. Chabchoub,,$^{1 *}$ N. Akhmediev, ${ }^{2}$ and N. P. Hoffmann ${ }^{1}$ \\ ${ }^{1}$ Mechanics and Ocean Engineering, Hamburg University of Technology, Eißendorfer Straße 42, 21073 Hamburg, Germany \\ ${ }^{2}$ Optical Sciences Group, Research School of Physics and Engineering, The Australian National University, Canberra ACT 0200, Australia
}

(Received 17 April 2012; published 11 July 2012)

\begin{abstract}
We present experimental results on the study of spatiotemporally localized surface wave events on deep water that can be modeled using the Peregrine breather solution of the nonlinear Schrödinger equation. These are often considered as prototypes of oceanic rogue waves that can focus wave energy into a single wave packet. For small steepness values of the carrier gravity waves the Peregrine breathers are relatively wide, thus providing an excellent agreement between the theory and experimental results. For larger steepnesses the focusing leads to temporally and spatially shorter events. Nevertheless, agreement between measurements and the Peregrine breather theory remains reasonably good, with discrepancies of modulation gradients and spatiotemporal symmetries being tolerable. Lifetimes and travel distances of the spatiotemporally localized wave events determined from the experiment are in good agreement with the theory.
\end{abstract}

DOI: 10.1103/PhysRevE.86.016311 PACS number(s): 05.45.Yv, 42.65.-k, 47.20.Ky, 92.10.Hm

\section{INTRODUCTION}

Several mechanisms for the formation of giant waves in the ocean, known as rogue waves, are presently under intense discussion [1-4]. One of the explanations is based on the linear theory of wave evolution. According to this approach, highamplitude waves appear as a result of constructive interference of many smaller-amplitude waves. The main deficiency of this approach is that when increasing the amplitude, the waves inevitably have to follow the laws of nonlinear dynamics. Indeed, one of the solid proofs of necessity of using nonlinear equations for surface gravity wave propagation was the experimental observation of modulation instability by Benjamin and Feir [5]. The most common approach to model the nonlinear dynamics of gravity waves in certain approximations is the nonlinear Schrödinger equation (NLS). Being relatively simple, it takes into account nonlinearity and linear dispersion. Despite relative simplicity, it describes well the phenomenon of Benjamin-Feir instability and highly nontrivial subsequent nonlinear wave dynamics [6-8]. Several experimental works confirmed validity of NLS for deep water waves [9-11]. In particular, Lake et al. [9] have shown that a wave with unstable periodic modulation evolves to near recurrence just like the NLS predicts.

One of the clear advantages of using the NLS is its integrability [12]. Having solutions in analytic form is attractive when comparing experimental results with theory. Indeed, the wellknown solution in the form of a traveling envelope soliton has been investigated in detail experimentally in Refs. $[8,11,13]$. Another class of solutions is breathers. One of the forms of breathers is solitary waves on finite amplitude background $[1,14,15]$. Due to nonlinear interference of the soliton with the finite background these solitons are pulsating. Surprising results of the theory are the breathers that pulsate only once. Among them is the Peregrine soliton [16] that is localized both in time and space. It is given by a rational expression and can be considered as the limiting case of either the space periodic breathers $[17,18]$ or the time periodic breathers $[19,20]$. Being

*amin.chabchoub@tuhh.de doubly localized, the Peregrine breather describes a unique wave event in which the waves of large amplitude seem to appear from nowhere and disappear without a trace [21]. As such, it has long been speculated to play a key role in rogue wave formation in the open ocean [22].

In contrast to solitons, experimental studies of breather solutions started only in recent years. After the first successful observation in nonlinear optics [23], the Peregrine breather has been observed in a water wave tank [24], in the case of surface gravity waves and later on for waves in multicomponent plasma [25]. In each case, remarkable agreement between the experiment and the Peregrine solution of the NLS has been found. The objective of the present work is to provide a detailed study of the rogue wave phenomenon based on breather theory. Here, we use a wide range of parameters of the background carrier wave and estimate the limits of applicability of breather theory. The paper is organized as follows: First, we discuss the Peregrine soliton solution of the NLS. Second, we describe the experimental approach on exciting and measuring wave states in the water wave tank. Then, we present the experimental results for various parameters of the background carrier wave. Additionally, we measured the lifetimes and travel distances of the spatiotemporally localized wave states and compared them with predictions of the NLS model.

\section{MATHEMATICAL APPROACH AND EXPERIMENTAL SETUP}

Weakly nonlinear deep water waves can be described by the NLS [7]:

$$
i\left(\frac{\partial a}{\partial t}+c_{g} \frac{\partial a}{\partial x}\right)-\frac{\omega_{0}}{8 k_{0}^{2}} \frac{\partial^{2} a}{\partial x^{2}}-\frac{\omega_{0} k_{0}^{2}}{2}|a|^{2} a=0,
$$

where $t$ and $x$ are time and space coordinates, while $k_{0}$ and $\omega_{0}=\omega\left(k_{0}\right)$ denote the wave number and the angular frequency of the carrier wave, respectively. The frequency $\omega_{0}$ and the wave number $k_{0}$ are linked by the dispersion relation of linear deep water wave theory, $\omega_{0}=\sqrt{g k_{0}}$, where $g$ denotes the gravitational acceleration. Accordingly, the group velocity of a wave packet is $c_{g}:=\left.\frac{d \omega}{d k}\right|_{k=k_{0}}=\frac{\omega_{0}}{2 k_{0}}$, which is half the phase 
speed of the carrier. The surface elevation $\eta(x, t)$ of the sea surface is then, to lowest order, given by

$$
\eta(x, t)=\operatorname{Re}\left(a(x, t) \exp \left[i\left(k_{0} x-\omega_{0} t\right)\right]\right) .
$$

A rescaled form of the NLS [12],

$$
i q_{T}+q_{X X}+2|q|^{2} q=0
$$

is obtained from (1) using the variables

$$
T=-\frac{\omega_{0}}{8 k_{0}^{2}} t, \quad X=x-c_{g} t=x-\frac{\omega_{0}}{2 k_{0}} t, \quad q=\sqrt{2} k_{0}^{2} a
$$

Here, $X$ is the coordinate in the frame moving with the wave group velocity and $T$ is the rescaled time. A family of space-periodic solutions of (3), which starts from a plane wave at $T \rightarrow-\infty$ and returns back to the plane wave when $T \rightarrow+\infty$, has been found in Refs. [17,18]:

$$
q_{A}(X, T)=\frac{\cosh (\Omega T-2 i \varphi)-\cos (\varphi) \cos (p X)}{\cosh (\Omega T)-\cos (\varphi) \cos (p X)} e^{2 i T} .
$$

Here $\Omega=2 \sin (2 \varphi), p=2 \sin (\varphi)$, and $\varphi \in \mathbb{R}$. It can be shown [18] that Eq. (4) is an exact solution of the NLS that represents growth-return cycles of modulation instability.

A solution describing a soliton on a background, which is periodic in time and tends to the plane wave solution as $X \rightarrow \pm \infty$, has been derived in Refs. [19,20]

$$
q_{M}(X, T)=\frac{\cos (\Omega T-2 i \varphi)-\cosh (\varphi) \cosh (p X)}{\cos (\Omega T)-\cosh (\varphi) \cosh (p X)} e^{2 i T},
$$

with $\Omega=2 \sinh (2 \varphi), p=2 \sinh (\varphi)$, and $\varphi \in \mathbb{R}$.

The solution first presented by Peregrine [16] can be understood as a limiting case of either of the above solutions when $\varphi \rightarrow 0$, i.e., when the spatial or the temporal period becomes infinite. The solution then takes rational form as a fraction of two polynomials:

$$
q_{P}(X, T)=\left(1-\frac{4(1+4 i T)}{1+4 X^{2}+16 T^{2}}\right) e^{2 i T} .
$$

Thus

$$
q_{P}(X, T)=\lim _{\varphi \rightarrow 0} q_{A}(X, T)=\lim _{\varphi \rightarrow 0} q_{M}(X, T) .
$$

The Peregrine solution breathes only once as it is localized in both space and time as shown in Fig. 1. The fact that makes this particular solution a prototype of a rogue wave is that the maximum amplification at the point of highest amplitude is three.

The experiments have been performed in a $15 \times 1.6 \times$ $1.5 \mathrm{~m}$ water wave tank with $1 \mathrm{~m}$ water depth. A single vertical flap activated by a hydraulic cylinder is located at one end of the tank and an absorbing beach is installed at the other end to avoid wave reflections. The surface height of the water at a given position is measured by a capacitance wave gauge with a sensitivity of $1.06 \mathrm{~V} / \mathrm{cm}$ which allows us to make measurements with an accuracy of up to three significant digits. The sampling frequency is $500 \mathrm{~Hz}$. The wave gauge is shown in Fig. 2, slightly to the left from the center of the tank.

In order to generate a Peregrine soliton solution of the NLS in the water wave tank, we first have to represent its analytical

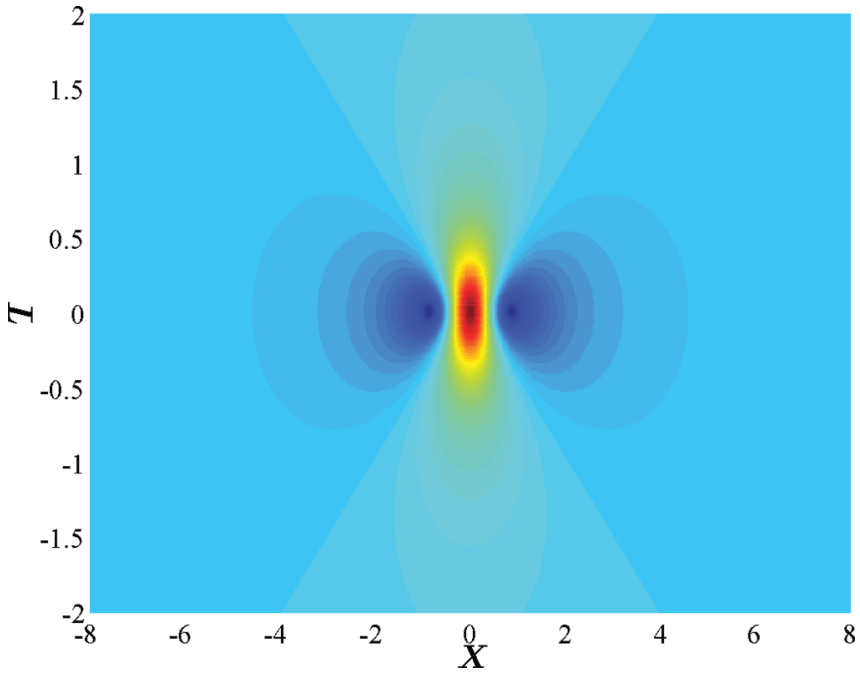

FIG. 1. (Color online) Contours of equal modulus of the Peregrine breather solution (6). The maximum amplitude occurs at $X=T=0$ and is exactly three times the amplitude of the background carrier wave.

expression in dimensional form relevant to Eq. (1):

$$
\begin{aligned}
q_{P}(x, t) & \\
= & a_{0}\left(1-\frac{4\left(1-i k_{0}^{2} a_{0}^{2} \omega_{0} t\right)}{1+\left[2 \sqrt{2} k_{0}^{2} a_{0}\left(x-c_{g} t\right)\right]^{2}+k_{0}^{4} a_{0}^{4} \omega_{0}^{2} t^{2}}\right) \\
& \times \exp \left(-\frac{i k_{0}^{2} a_{0}^{2} \omega_{0}}{2} t\right),
\end{aligned}
$$

and take into account the fact that to lowest order the surface elevation is then given by

$$
\eta(x, t)=\operatorname{Re}\left\{q_{P}(x, t) \exp \left[i\left(k_{0} x-\omega_{0} t\right)\right]\right\} .
$$

In this approximation, we are ignoring the bounded waves. Equation (8) is used to determine the initial conditions for the flap motion and to compare subsequent measured data with NLS-based theoretical prediction.

The range of parameters for the carrier wave is limited in the experiment by a number of factors. First of all, due to the limited length of the tank, wavelength has to be chosen sufficiently short to observe both growth and decay processes. Conversely, the wavelength needs to be large enough to ignore the effects of surface tension. When the tank size is given, the wavelength should also satisfy the conditions of deep water. Namely, the depth $d$ and the wave number $k_{0}$ should satisfy the condition $k_{0} d \gg 1$ [26]. When taking into account all these requirements, the range of wavelengths that can be excited in the tank is becoming very narrow. The actual numbers used in the experiments are given below.

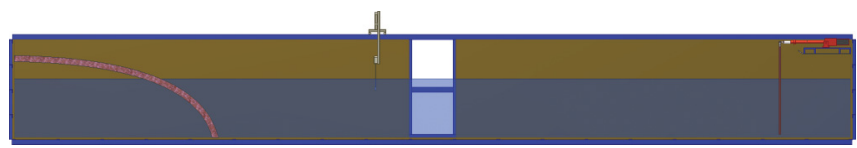

FIG. 2. (Color online) Schematic illustration (side view) of the water wave tank. The beach is shown at the left, the paddle at the right. The capacitance wave gauge is movable along the tank. 
Thus, before starting the experiment, we determine the initial amplitude and the frequency of the carrier in order to satisfy deep water conditions. As a preliminary exercise, we generate the harmonic wave with the specific constant amplitude along the tank in centimeter scale. Once generated, the amplitude is almost constant along the whole tank, except for the small region near the flap. They are nearly constant up to the beach, which is a signature of small dissipation in the experiment. By generating waves with various amplitudes, we established that within the small range we are using the wave amplitudes are linearly proportional to the signal which determines the flap motion. The coefficient of proportionality is adjusted empirically. This preliminary procedure allows us to determine the scaling factor to a chosen amplitude $a_{0}$ in the above equations. The wave number $k_{0}$ is derived from the linear dispersion relation. After these preparations, we are ready to use more complicated initial conditions. In particular, a simple negative translation of Eqs. (8) and (7) along the $x$ axis provides us with the initial condition that should be applied to the flap in order to observe the Peregrine soliton.

\section{EXPERIMENTAL RESULTS}

\section{A. Spatiotemporal growth and decay}

In order to demonstrate the existence of Peregrine solitons, we performed a number of experiments with different wave numbers and amplitudes of the carrier wave. An illustrative example is shown in Fig. 3. Here, the amplitude of the background is chosen to be $a_{0}=0.005 \mathrm{~m}$, the carrier frequency $f_{0}=2.40 \mathrm{~Hz}$, or $\omega_{0}=15.1 \mathrm{~s}^{-1}$, the wave number $k_{0}=23.2 \mathrm{~m}^{-1}$, and the wavelength $\lambda_{0}=0.270 \mathrm{~m}$. Thus, the steepness of this carrier wave becomes $\varepsilon:=a_{0} k_{0}=0.116$.

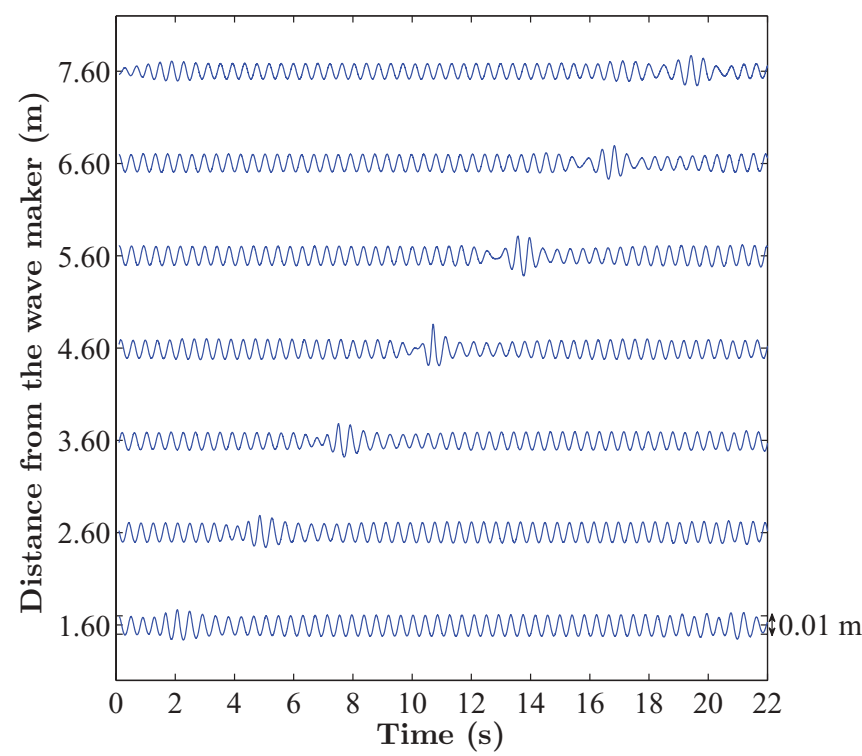

FIG. 3. (Color online) Illustration of the spatiotemporal evolution of the water surface height at various distances from the wave maker. The curves are measured at distances from the wave maker shown in the vertical axis on the left. Surface elevation for each curve is measured in centimeters, as shown in the vertical axis on the right. Parameters chosen for the experiment are $a_{0}=0.005 \mathrm{~m}$, $k_{0}=23.2 \mathrm{~m}^{-1}$; consequently, $\varepsilon:=a_{0} k_{0}=0.116$.
The flap motion was programmed to generate a wave with the maximum breather amplitude at a distance $4.60 \mathrm{~m}$ along the tank. Water surface elevation measurements have been collected at nine positions, with equal separations of $1 \mathrm{~m}$ along the direction of wave propagation. These data show that the carrier wave that is slightly modulated near the flap propagates along the tank with the group velocity. Small perturbation of the carrier wave grows such that at about $4.60 \mathrm{~m}$ along the tank the amplitude reaches its maximum with an amplification factor of three. In good agreement with the NLS theory, afterward the amplitude decays. Thus, if we forget for a moment that initial conditions are created by ourselves, the rogue wave on top of the carrier wave appears seemingly out of nowhere.

In the sections that follow, we first explore the influence of carrier parameters on wave evolution. This allows us to evaluate the extent of agreement between measurements and theoretical predictions of the NLS as well as its limitations. Then, we give further quantitative evaluation of the spatiotemporal properties of Peregrine breathers in terms of lifetimes and travel distances.

\section{B. The influence of carrier wave steepness}

The analytical expression of the Peregrine breather in dimensional form [Eqs. (7) and (8)] suggests that at the position where the maximum wave amplitude is achieved, for a given wave frequency $\omega_{0}$, the time evolution depends, apart from an amplitude scaling, only on the steepness $\varepsilon=a_{0} k_{0}$ of the carrier wave. We therefore performed a number of experiments to study to what extent this parameter influences the measured data.

Figure 4 shows the results for three different amplitudes of the carrier: $0.010,0.020$, and $0.030 \mathrm{~m}$. The wavelength of the carrier has been adapted for each measurement to yield a wave steepness $\varepsilon=0.116$. The point of wave maximum has been chosen $9 \mathrm{~m}$ away from the wave maker. We can see, from Fig. 4, that within the amplitude range considered, the resulting time series do scale quite well. The noticeable phase

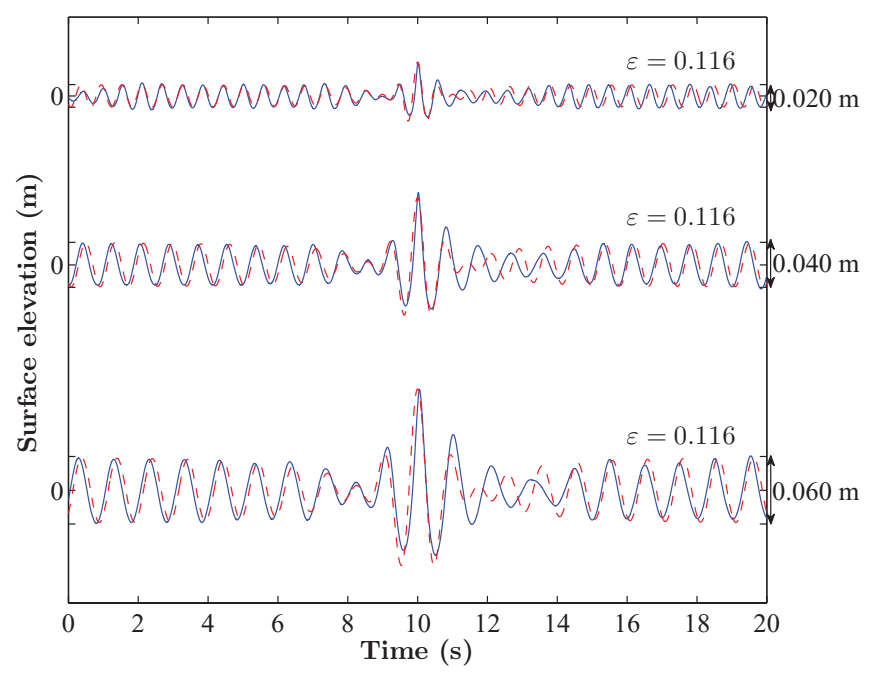

FIG. 4. (Color online) Comparison of measured (solid line) wave heights at the position of maximum wave amplitude with Peregrine solution (dashed line) evaluated at $X=0$. 


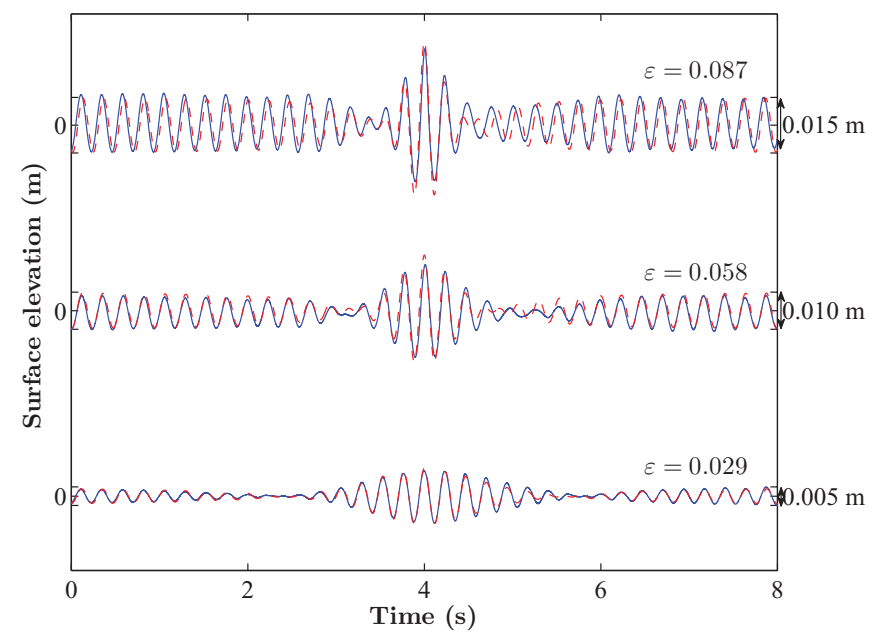

FIG. 5. (Color online) Comparison of measured (solid line) wave profiles at the position of maximum wave amplitude with theoretical Peregrine soliton solution (dashed line) evaluated at $X=0$ for steepness $0.087,0.058$, and 0.029 by varying the amplitude. The wave number $k_{0}=11.6 \mathrm{~m}^{-1}$.

shift to the right of the maximum amplitude may be related to higher steepness in the middle of the wave train and the corresponding influence of higher Stokes harmonics, which we ignored in Eq. (8).

Figures 5 and 6 show the influence of the wave steepness of the carrier wave on the resulting surface elevation at the point of maximum amplitude. To vary the steepness, we can alter either carrier amplitude or carrier wave number, or both simultaneously. First, we fix the the value of $k_{0}=11.6 \mathrm{~m}^{-1}$ while the amplitudes are varied in order to obtain the steepness values of $0.087,0.058$, and 0.029 , respectively. The results for the three cases are shown in Fig. 5. In another set of experiments we fixed the amplitude at the level $a_{0}=0.010 \mathrm{~m}$ while the wavelength of the carrier is varied in a way to obtain

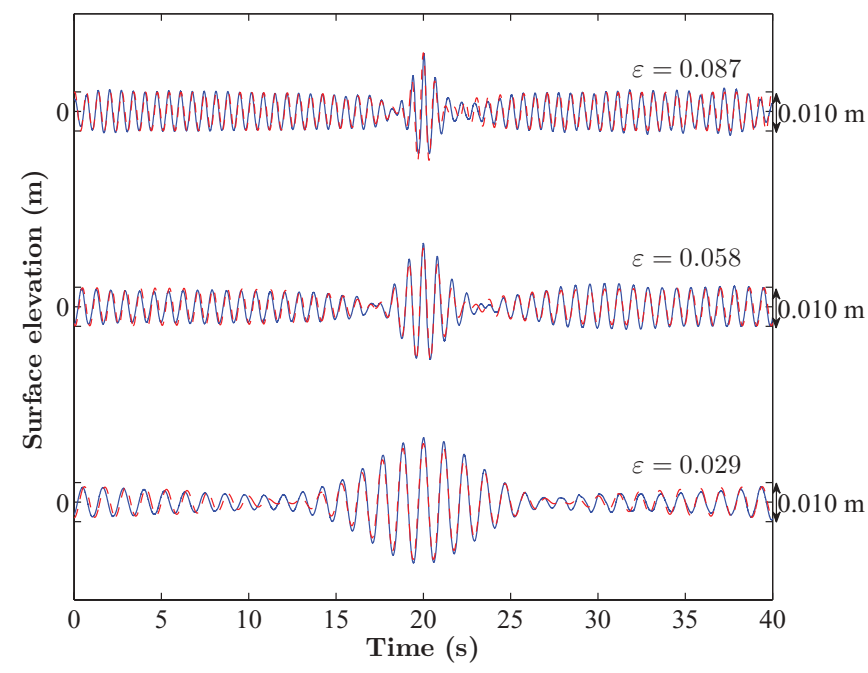

FIG. 6. (Color online) Comparison of measured surface heights at the position of maximum wave amplitude (solid line) with Peregrine soliton solution (dashed line) evaluated at $X=0$ for steepness 0.087 , 0.058 , and 0.029 by varying the wave number. The scaling parameter $a_{0}=0.010 \mathrm{~m}$. the same steepness values as before. The results of this set are shown in Fig. 6. For all measurements, the experimental data are compared to the analytical Peregrine solution.

A few important conclusions can be drawn from the above results. First, in all measurements, the maximum amplitude amplification of three is reached in perfect accordance with the theoretical prediction. Second, the number of waves within the wave packet increases strongly with decreasing steepness $\varepsilon$. This fact is also in very good agreement with NLS-based prediction. Third, the agreement between the measurement and the NLS theory is higher for smaller steepness values. This is consistent with the assumption of a weakly nonlinear nature of the NLS theory, which is valid for small steepness values. For larger steepness values, discrepancies between the theory and the experiment start to appear. To some extent, these discrepancies have been noticed in Ref. [24]. We can also notice here that the gradients in amplitude modulation are smaller in theory than in experiment. There is also a noticeable asymmetry in the wave evolution when comparing the wave profile before and after the point of maximum amplitude. In addition to these discrepancies, our measurements confirm that the wave steepness of the carrier is one of the decisive parameters in the experiment.

In order to understand the increased number of waves within the packet, we recall some results known from Benjamin-Feir instability and Akhmediev breathers. The band of unstable wave numbers $K$ according to Benjamin-Feir is

$$
0<K<2 \sqrt{2} k_{0}^{2} a_{0}
$$

(see, e.g., [15] or [8]). The theory allows us to connect the amplitude amplification of Akhmediev breathers to the steepness and number of waves under the resulting modulations $[15,27,28]$ :

$$
\begin{aligned}
\frac{a_{\max }}{a_{0}} & =1+2 \sqrt{1-\left(\frac{K}{2 \sqrt{2} k_{0}^{2} a_{0}}\right)^{2}} \\
& =1+2 \sqrt{1-\left(\frac{1}{2 \sqrt{2} N_{x} \varepsilon}\right)^{2}}
\end{aligned}
$$

where $N_{x}:=\frac{\lambda_{\bmod }}{\lambda_{0}}=\frac{k_{0}}{K}$ is the number of waves in one period of modulation in the space series and $\varepsilon=a_{0} k_{0}$ is the steepness of the carrier. According to the linear dispersion relation, we have

$$
N_{t}=2 N_{x}
$$

where $N_{t}$ denotes the number of modulated waves in a corresponding time series. Thus, Eq. (10) becomes

$$
\frac{a_{\max }}{a_{0}}=1+2 \sqrt{1-\left(\frac{1}{\sqrt{2} N_{t} \varepsilon}\right)^{2}} .
$$

For each Akhmediev breather (4) determined by a fixed value of the parameter $\varphi$, there is a direct connection between the number of modulated waves in time series $N_{t}$ and the steepness of the carrier wave. Only the product of $N_{t}$ and $\varepsilon$ enters the above equation, and for given amplification factors, large steepness leads to a small number of waves and vice versa. The Peregrine breather, considered in the present study, 


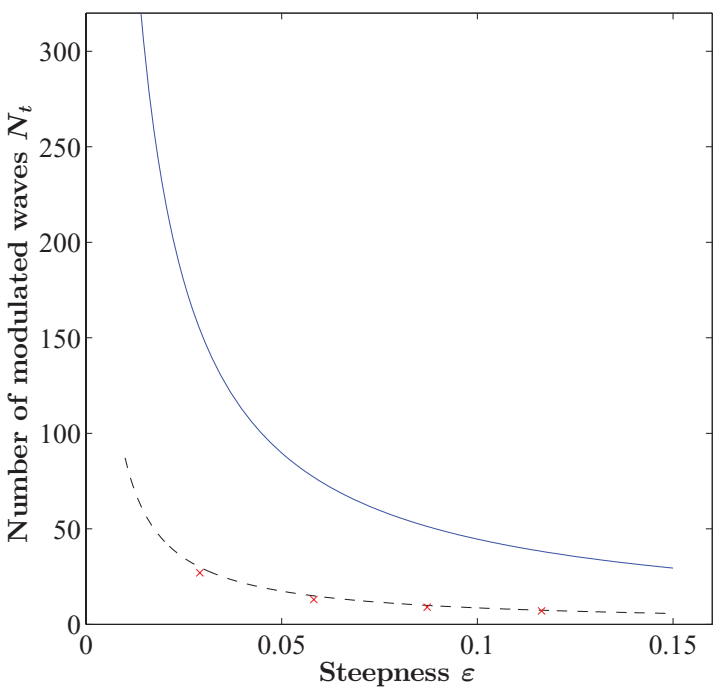

FIG. 7. (Color online) The number of modulated waves in time $N_{t}$ vs steepness $\varepsilon$. The value of $N_{t}$ calculated for the Peregrine breather when the threshold amplitude is 0.99 (solid line) or 0.75 (dashed line). Crosses $\times$ represent the experimental values obtained for a threshold amplitude of 0.75 .

has a maximum amplification of exactly three and can be obtained from the Akhmediev breather family by taking the limit $K \rightarrow 0$ within the Benjamin-Feir instability band.

The analytical formulas above do not provide the functional relationship between $N_{t}$ and $\varepsilon$. Nevertheless, such a relationship can be found numerically. We calculated the dependance of $N_{t}=\frac{T_{\bmod }}{T_{0}}$ on $\varepsilon$ for the Peregrine breather, where $T_{\bmod }$ and $T_{0}$ denote the period of the modulation and the period of the carrier, respectively. For this calculation, we chose a threshold value of 0.99 times the wave amplitude to define the points where modulation appears or disappears. This choice resulted in reasonable agreement with the asymptotic behavior. On the other hand, in order to compare the theoretical predictions with experimental data, which always contain noise, we chose a more robust threshold of 0.75 times the wave amplitude. Figure 7 shows each of these results. As can be seen from the figure, for the Peregrine breather, the number of modulated waves is roughly inversely proportional to the wave steepness. A similar result has been found by [16] and [29]. We can also see that our experiments (crosses $x$ ) show good agreement with the predictions of the NLS theory.

\section{LIFETIMES AND TRAVEL DISTANCES}

The major feature of the Peregrine breather is its maximum surface elevation reached at a single point. In contrast to other solitonlike solutions which decay exponentially out of the region of localization, the Peregrine breather experiences a growth-decay cycle described by rational functions. This means that this formation has weaker spatial and temporal localization. In order to describe quantitatively the degree of localization, we can introduce the breather's "lifetime" and "travel distance." These quantities can be defined in various ways. The idea is to introduce certain temporal and spatial intervals where the amplitude has significant deviation from the homogeneous background. One of the possibilities is to define the time or space intervals such that at the edges of these intervals the wave has a fixed threshold amplitude, which is a specified fraction of the maximum amplitude value. Below, we calculated these intervals based on the analytical solution and also compared the results with measurements.

\section{A. Laboratory scale}

As the threshold amplitude is somewhat arbitrary, we used three different values in order to see if it is crucial for
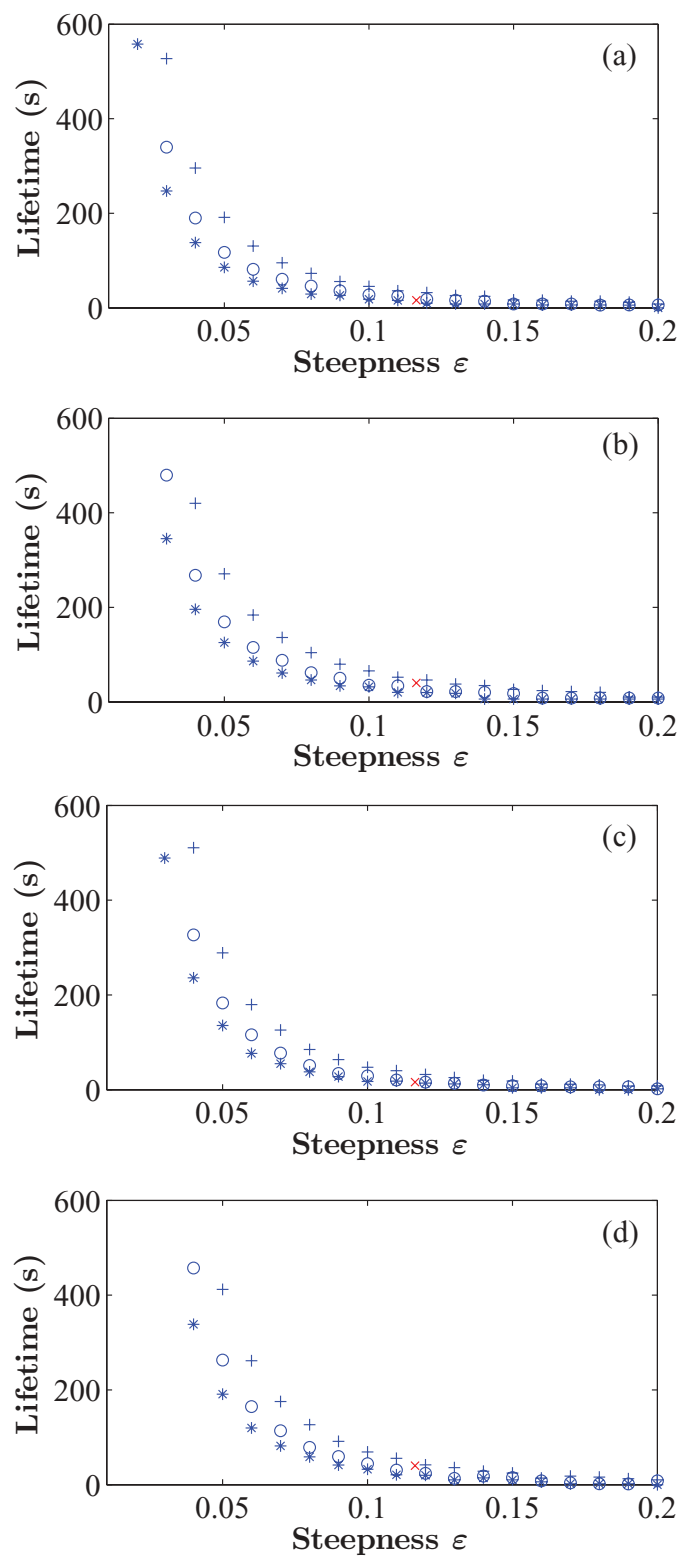

FIG. 8. (Color online) Lifetimes of Peregrine breather vs steepness, calculated for the amplitude thresholds of 1.25 (crosses), 1.50 (circles), and 1.75 (stars). Four cases correspond to the following choice of parameters: (top panel) $k_{0}=23.2 \mathrm{~m}^{-1}$; (second panel) $k_{0}=11.6 \mathrm{~m}^{-1}$; (third panel) $a_{0}=0.005 \mathrm{~m}$; and (bottom panel) $a_{0}=$ $0.010 \mathrm{~m}$. Crosses $\times$ denote experimental results for the amplitude threshold of 1.75 [(a) and (c)] and 1.25 [(b) and (d)]. 

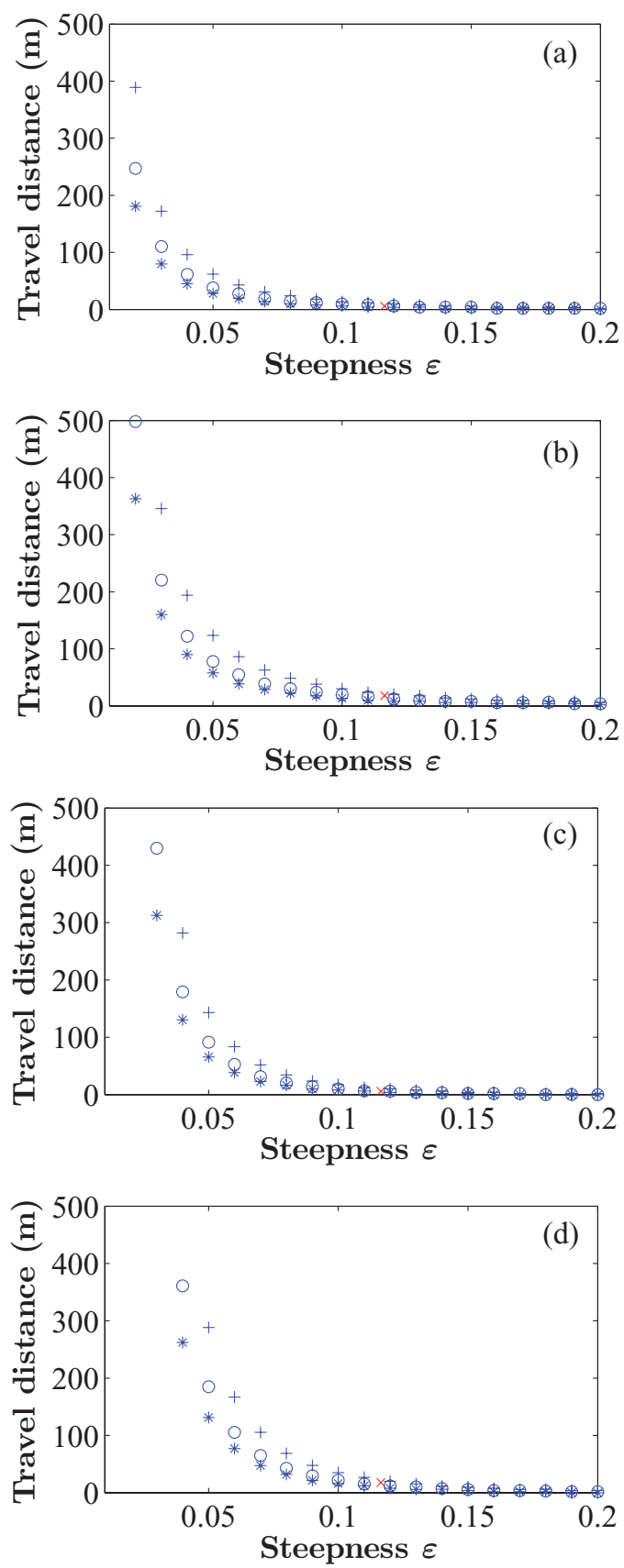

FIG. 9. (Color online) Travel distances of Peregrine breather vs steepness, calculated for the amplitude thresholds of 1.25 (crosses), 1.50 (circles), and 1.75 (stars). Four cases correspond to the following choice of parameters: (top panel) $k_{0}=23.2 \mathrm{~m}^{-1}$; (second panel) $k_{0}=11.6 \mathrm{~m}^{-1}$; (third panel) $a_{0}=0.005 \mathrm{~m}$; and (bottom panel) $a_{0}=$ $0.010 \mathrm{~m}$. Crosses $\times$ denote experimental results for the amplitude threshold of 1.75 [(a) and (c)] and 1.25 [(b) and (d)].

our definitions. Namely, we used the thresholds $1.25,1.50$, and 1.75 for exceeding the envelope over the background at the edges of the intervals. We recall that at the point of maximum, the envelope exceeds three times the background. Figures 8 and 9 show the results of calculations as well as experimental results (crosses $\times$ ). In dimensional units, there are a few parameters that influence the lifetime and the travel distance. These are the amplitude, wave number,
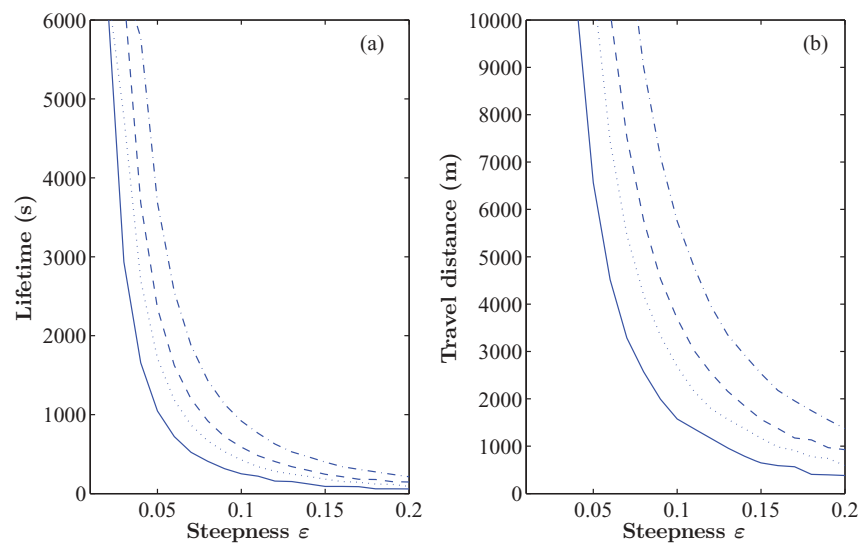

FIG. 10. (Color online) Lifetimes (a) and travel distances (b) of Peregrine-type wave structures vs steepness for $\lambda=100 \mathrm{~m}$, defined by amplification factors of 1.25 (dash-dotted), 1.50 (dashed), 1.75 (dotted), and 2.20 (solid).

frequency, and steepness. The plots show the lifetime vs wave steepness presented for several values of the wave number and frequency. As before, to vary the steepness, we either changed the wave number keeping the amplitude fixed, or we varied the amplitude keeping the wave number fixed. Direct measurement of lifetimes and travel distances is difficult since the length of the wave tank is limited. These data have to be calculated from the measurements of the wave profiles. This way, two data points have been produced, one based on the results shown in Fig. 3 above $\left(k_{0}=23.2 \mathrm{~m}^{-1}\right)$ and the other point based on the results obtained in Ref. [24] $\left(k_{0}=11.6 \mathrm{~m}^{-1}\right)$.

As expected, travel distances and lifetimes of the Peregrine soliton decrease quickly with the increase of the wave steepness. The experimentally obtained values shown by crosses $\times$ fit well to the NLS-based predictions for corresponding threshold values 1.75 (shown by stars) or 1.25 (shown by crosses), respectively. We assume that dissipation can be ignored within the growth-decay cycle of the Peregrine breather.

\section{B. Ocean scale}

For illustration purposes we calculated lifetimes and travel distances for the case of ocean waves with a wavelength of $\lambda_{0}=100 \mathrm{~m}$. In addition to the threshold amplification factors given above, we also used the amplification factor of 2.2, which is still smaller than the maximum amplification of three. This factor is justified if we recall that the ocean waves exceeding the background wave height (roughly speaking, the significant wave height) by a factor of 2.2 are already rogue waves. Thus, the Peregrine breather can be a considered as an ocean rogue wave within extended intervals in time and space.

Figure 10 shows the results. We can see, from this figure, that for particular wave steepness values of the order 0.100 , the lifetime of a Peregrine breather would be of the order of a few minutes, while its travel distance is of the order of a few kilometers. Although being very short in the scale of the world ocean, these numbers are still sufficiently long if we intend to develop early warning systems directly installed on ships. 


\section{CONCLUSIONS AND FUTURE DIRECTIONS}

Our study shows that the Peregrine solution of the NLS equation which models regular deep water surface gravity waves can be observed experimentally in a water wave tank. It can be generated directly by applying initial-boundary conditions derived from the exact solution of the NLS. In the weakly nonlinear regime, when the steepness of the underlying background carrier wave is small, the agreement between experimental results and the Peregrine solution of the NLS is very good. Discrepancies start to grow with increasing the steepness values. The carrier wave steepness is also a key parameter with respect to the spatiotemporal wave shape. At higher steepnesses the resulting wave packets become very narrow in space and time. Consequently, the lifetime and travel distance of the spatiotemporally localized wave structures decrease.

Although our results demonstrate reasonably good correlation between the wave tank measurements and the dynamics of the NLS solution, they also clearly show limitations of the NLS in the present context. When nonlinearity becomes stronger, i.e. steepness increases, the measured waves become asymmetric. The modulation gradients also become larger than those suggested by NLS theory. The origin of these discrepancies deserves further study. One way to improve the modeling is to use higher-order envelope equations offered, e.g., by Dysthe [30] or Slunyaev [31]. Wave breaking that can be observed for even higher carrier steepness is another limitation that needs attention. In addition, for ocean waves, the background wave state is irregular. The role of Peregrine breathers in such a natural sea state has to be studied separately. Work in this direction has already started.

\section{ACKNOWLEDGMENTS}

A.C. would like to thank Eivind Gard Lund for helpful discussions on numerics and Miguel Onorato for discussions concerning the experiments. N.A. and N.P.H. acknowledge the support of the Volkswagen Stiftung. N.A. acknowledges partial support of the Australian Research Council (Discovery Project DP110102068). N.A. acknowledges support through an Alexander von Humboldt Award.
[1] C. Kharif, E. Pelinovsky, and A. Slunyaev, Rogue Waves in the Ocean (Springer, Heidelberg, 2009).

[2] C. Garrett and J. Gemmrich, Phys. Today 62(6), 62 (2009).

[3] P. Müller, C. Garrett, and A. Osborne, Oceanography 18, 66 (2005).

[4] M. Onorato, A. Osborne, M. Serio, and S. Bertone, Phys. Rev. Lett. 25, 5831 (2001).

[5] T. B. Benjamin and J. E. Feir, J. Fluid Mech. 27, 417 (1967).

[6] M. J. Lighthill, J. Inst. Math. Appl. 1, 269 (1965).

[7] V. Zakharov, J. Appl. Mech. Tech. Phys. 9, 190 (1968).

[8] H. C. Yuen and B. M. Lake, Adv. Appl. Mech., 22, 67 (1982).

[9] B. M. Lake, H. C. Yuen, H. Rungaldier, and W. E. Ferguson, J. Fluid Mech. 83, 49 (1977).

[10] H. C. Yuen and W. E. Ferguson, Phys. Fluids 21, 1275 (1978).

[11] H. C. Yuen and B. M. Lake, Phys. Fluids 18, 956 (1975).

[12] V. E. Zakharov and A. B. Shabat, Sov. Phys. JETP 34, 62 (1972).

[13] B. M. Lake and H. C. Yuen, J. Fluid Mech. 83, 75 (1977).

[14] K. B. Dysthe and K. Trulsen, Phys. Scr., T 82, 48 (1999).

[15] A. Osborne, Nonlinear Ocean Waves and the Inverse Scattering Transform (Elsevier, New York, 2010).

[16] D. H. Peregrine, J. Aust. Math. Soc. Series B, Appl. Math. 25, 16 (1983).

[17] N. Akhmediev, V. M. Eleonskii, and N. Kulagin, Zh. Eksp. Teor. Fiz. 98, 1542 (1985) [Sov. Phys. JETP 61, 894 (1985)].
[18] N. Akhmediev and V. I. Korneev, Theor. Math. Phys. 69, 1089 (1986).

[19] E. A. Kuznetsov, Dok. (USSR) 236, 575 (1977) [Sov. Phys. Dokl. 22, 507 (1977)].

[20] Y. C. Ma, Stud. Appl. Math. 60, 43 (1979).

[21] N. Akhmediev, A. Ankiewicz, and M. Taki, Phys. Lett. A 373, 675 (2009).

[22] V. I. Shrira and V. V. Geogjaev, J. Eng. Math. 67, 11 (2010).

[23] B. Kibler, J. Fatome, C. Finot, G. Millot, F. Dias, G. Genty, N. Akhmediev, and J. M. Dudley, Nat. Phys. 6, 790 (2010).

[24] A. Chabchoub, N. P. Hoffmann, and N. Akhmediev, Phys. Rev. Lett. 106, 204502 (2011).

[25] H. Bailung, S. K. Sharma, and Y. Nakamura, Phys. Rev. Lett. 107, 255005 (2011).

[26] C. C. Mei, The Applied Dynamics Of Ocean Surface Waves (Wiley, New York, 1983).

[27] M. Onorato, A. Osborne, M. Serio, and T. Damiani, in Proceedings of the Workshop "Rogue Waves 2000," edited by M. Olagnon and G. Athanassoulis (IFREMER, Brest, 2000), pp. 181-191.

[28] M. Onorato, D. Proment, and A. Toffoli, Phys. Rev. Lett. 107, 184502 (2011).

[29] K. L. Henderson, D. H. Peregrine, and J. W. Dold, Wave Motion 29, 341 (1997).

[30] K. B. Dysthe, Proc. R. Soc. London, Sect. A 369, 105 (1979).

[31] A. Slunyaev, Zh. Eksp. i Teor. Fiz. 128, 1061 (2005) [J. Exp. Theor. Phys. 101, 926 (2005)]. 